\title{
A bidimensional model of pitch in the recognition of melodies
}

\author{
WENDY L. IDSON \\ University of Texas at Austin, Austin, Texas 78712 \\ and \\ DOMINIC W. MASSARO \\ University of Wisconsin at Madison, Madison, Wisconsin 53706
}

\begin{abstract}
Pitch can be conceptualized as a bidimensional quantity, reflecting both the overall pitch level of a tone (tone height) and its position in the octave (tone chroma). Though such a conceptualization has been well supported for perception of a single tone, it has been argued that the dimension of tone chroma is irrelevant in melodic perception. In the current study, melodies were subjected to structural transformations designed to evaluate the effects of interval magnitude, contour, tone height, and tone chroma. In two transformations, the component tones of a melody were displaced by octave intervals, either preserving or violating the pattern of changes in pitch direction (melodic contour). Replicating previous work, when contour was violated perception of the melody was severely disrupted. In contrast, when contour was preserved the melodies were identified as accurately as the untransformed melodies. In other transformations, a variety of forms of contour information were preserved, while eliminating information for absolute pitch and interval magnitude. The level of performance on all such transformations fell between the levels observed in the other two conditions. These results suggest that the bidimensional model of pitch is applicable to recognition of melodies as well as single tones. Moreover, the results argue that contour, as well as interval magnitude, is providing essential information for melodic perception.
\end{abstract}

Psychophysical investigations have focused upon developing unidimensional psychological scales of pitch as a monotonically increasing function of the unidimensional physical scale of frequency. Though such scales have been developed with reasonable success (e.g., Stevens \& Volkmann, 1940; Stevens, Volkmann, \& Newmann, 1937), there is a body of research to suggest that pitch perception may be more complex than a simple monotonic scale would indicate (Attneave \& Olson, 1971; Bachem, 1954; Baird, 1971; Deutsch, 1972b, 1973a, 1974; Shepard, 1964). Rather, it has been proposed that pitch can

This research was supported by U.S. Public Health Service Grant MH-19399 to D. W. Massaro, and was conducted while W. L. Idson was supported first by a University of Wisconsin Graduate Traineeship and later by a University of Wisconsin Graduate Fellowship. The work represents a collaborative effort; order of authorship is arbitrary. We would like to thank David E. Klitzke for his assistance with the computer programming. Portions of this work were discussed at the Meeting of the Acoustical Society of America, San Diego, 1976, and at the Meeting of the Midwestern Psychological Association, Chicago, 1977. Requests for reprints may be sent to either author: Wendy L. Idson at the Department of Psychology, University of Texas at Austin, Austin, Texas 78712; or Dominic W. Massaro at the Department of Psychology, University of Wisconsin, Madison, Wisconsin 53706. be more appropriately conceptualized as a bidimensional quantity representing both the overall pitch level of the tone (tone height) and its position in the octave (tone chroma) (Bachem, 1948; Deutsch, 1969; Meyer, 1904; Révész, 1954; Ruckmick, 1929; Shepard, 1964; Drobish, cited in Ruckmick, 1929). Most bidimensional descriptions share the assumptions embodied in the original model proposed by Drobisch (1846, cited in Ruckmick, 1929). Figure 1 shows a graphical representation of this model, in which pitch is conceptualized as a helix, with tone height represented by the vertical axis of the helix and tone chroma by the circular scale at its base. Tones separated by an integer number of octaves (a $2: 1$ frequency ratio) are located at corresponding points on successive turns of the helix.

A bidimensional model is implicit in the traditional musical scale, which is based on the underlying similarity of tones which stand in an octave relationship. Such tones share the same name (e.g., $A_{4}=435 \mathrm{~Hz}$, $A_{s}=870 \mathrm{~Hz}$ ). and in some cases are treated as harmonically equivalent (Tovey, 1956). For example, if the component tones of a chord are displaced into different octaves, this new chord will be treated as harmonically equivalent to the original chord. These similarities are not limited to Western music, the 


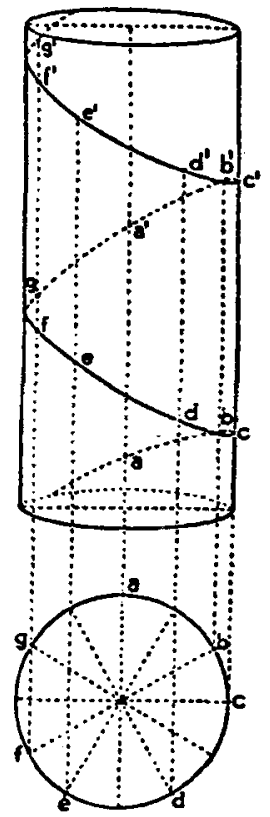

Figure 1. Graphic representation of a bidimensional model (taken from Révész, 1954).

octave relationship being fundamental in music from a variety of cultures (Nettle, 1956). This musical equivalence appears to be reflected at the perceptual level, as well. Both Bachem (1954) and Baird (1971) have found that while subjects with absolute pitch can almost invariably name single tones correctly, they are often unable to place them in the appropriate octave. For example, a subject might be aware that a particular tone was $C$, but would be unable to distinguish whether it was $\mathrm{C}_{4}, \mathrm{C}_{5}$, or $\mathrm{C}_{6}$.

If a bidimensional model is appropriate for pitch perception, then two tones which differ by an exact octave should be perceived as more similar than two tones which differ by less than an octave, despite the fact that the frequency separation is greater in the former case. Allen (1967) reported this result for the subjective ratings of tone pairs. Moreover, early conditioning work demonstrated enhanced generalization effects, both in rats (Blackwell \& Schlosberg, 1943) and humans (Humphreys, 1939), for tones at octave intervals. The most interesting evidence comes from a study by Shepard (1964). He reasoned that if one of the components of pitch was circular, then it should be possible to eliminate transitivity in pitch judgments by suppressing the other component. To eliminate the dimension of tone height, Shepard generated a set of 12 complex tones, each composed of pure tones separated by octave intervals. When the complex tones were sounded up (or down) the scale in half-note steps, at a rate of 1 tone/sec, the tones were heard as constantly ascending (or descending) in pitch. A series of complex tones, whose components were not at octave intervals, was heard appropriately as a sequence of chords.
Deutsch (1969) incorporated a bidimensional model of pitch into a more general model designed to account for the recognition of music. In Deutsch's model, the pitch of a tone is first stored in a unidimensional array of tone height. Processing then continues along one of two channels. Single tones will be analyzed for tone chroma, along a channel in which a convergence of pitch information occurs from all tones at octave intervals. The tones of a melody will be analyzed along the second channel, in which octave convergence is assumed not to occur. Instead, the pitch intervals between successive tones will be abstracted and synthesized, in accord with a hierarchical neural-network model analogous to that of Hubel and Wiesel (1962).

There are two principal consequences of Deutsch's (1969) model. The first, which would also be true for any bidimensional model, is that perception of single tones should be affected by tone chroma as well as tone height. The second maintains that perception of the tones of a melody should not be influenced by tone chroma. It should be stressed that this latter result is not a necessary consequence of a bidimensional model of pitch, but is unique to Deutsch's (1969) model.

In order to evaluate the first of these consequences, Deutsch $(1973 a, 1974)$ investigated the effects of tone chroma on the recognition of single tones. She employed a task in which subjects were to determine whether a comparison tone was the same as or different in pitch from a previously presented standard tone. Earlier work had demonstrated three specific effects in this task, when a series of interference tonesall drawn from a single octave-are interposed between the standard and comparison tones. First, if the sequence of interference tones is chosen randomly from within the octave, performance is decreased relative to a blank interval (Deutsch, 1970). Second, when the standard and comparison tones differ in frequency, including an interference tone which is identical to the comparison will disrupt performance more greatly than a randomly selected tone (Deutsch, $1970,1972 a)$. Third, an even larger performance decrement is found when the standard and comparison tones are identical in frequency and the sequence of interference tones includes one a semitone higher and another a semitone lower than the standard/. comparison (Deutsch, 1973b). In a series of studies in which the critical tone(s) were displaced by octave intervals, Deutsch $(1973 a, 1974)$ was able to replicate all three of these effects, though their magnitude was somewhat smaller. These results provide strong support for the bidimensional model. The occurrence of interference from tones drawn from the higher and lower octaves argues for the significance of tone chroma, while the decrement in the magnitude of the interference, relative to interference tones in the same octave, supports the contribution of tone height. 
Deutsch (1972b) also attempted to provide support for the second prediction of her model, by demonstrating simultaneously that octave convergence will not occur for the component tones of melodies and that successive interval abstraction provides the basis for melodic perception. If octave convergence occurs for melodies, then displacing the component tones of a melody by octave intervals should not disrupt recognition; the pitch information provided by tone chroma should be sufficient. If, however, melodic perception is based upon successive intervals and octave convergence does not occur, perception should be disrupted, as displacing the tones by octave intervals will eliminate tone height and distort successive intervals. To evaluate this hypothesis, Deutsch presented subjects with the first half of the tune Yankee Doodle, played either entirely in one of three adjacent octaves or with the component tones displaced by octave intervals, randomly throughout the three adjacent octaves. Although the melody was easily identified when it was played entirely in any one of the three octaves, subjects were completely unable to recognize the melody when the tones were randomly displaced among the three octaves. On the basis of these results, Deutsch (1972b) argued that octave convergence does not occur for melodies and that successive intervals provide the critical information for melodic perception. This latter conclusion is based upon the fact that the octave displacement manipulation primarily distorts information for tone height and successive intervals. Yet tone height does not appear to be critical, as the melody could be recognized when it was played entirely in any of three octaves. Deutsch concluded that the critical information which is eliminated by octave displacement comes from the magnitude of successive pitch intervals.

The intent of the present research is to argue that rejection of a bidimensional model of pitch for the perception of melodies may be premature. The reason that is generally given for rejecting a bidimensional model is that melodies are perceived as a string of successive intervals. If intervals are computed between the absolute frequencies (tone height) of the component tones, then the octave similarities offered by the chroma dimension cannot be useful. For example, the absolute magnitude of the interval $\mathrm{C}_{4}$ to $D_{5}$ is clearly larger than that of $C_{4}$ to $D_{4}$, minimizing the similarity between them. Yet a bidimensional model can be conceptualized in such a way that it avoids this consequence. If a convergence of pitch information occurs from tones standing in an octave relationship, then it seems tenable that all such tones may be collapsed into a single abstract representation. Thus, there would be one abstract $A$ representing all As, one abstract A\# representing all A\#s, out to one abstract $G \#$ representing all $G \#$ s. Perception of a melody could then occur within the resulting abstract octave, represented by the circle in Figure 1. Under such a scheme, the intervals $C_{4}$ to $D_{4}$ and $C_{4}$ to $D_{5}$ would be equivalent, both simply representing the 2-semitone interval from $C$ to $D$. Within such a framework, tone chroma could play as great a role in the perception of melodies as in the perception of single tones. While such a conceptualization is not a necessary correlate of a bidimensional model of pitch, it is certainly a viable one, suggesting that in no sense are the concepts of successive interval abstraction and tone chroma theoretically irreconcilable.

Yet it might be asked whether such a conceptual framework should not predict that subjects would be able to recognize the distorted versions of Yankee Doodle in Deutsch's (1972b) study, as all of the tones would converge into a single octave space. However, Deutsch's manipulation effectively eliminated the potential utility of an abstract octave. In displacing the component tones of the melody across octaves, Deutsch made no attempt to preserve the directions of the successive intervals in the original melody. It might be expected that the direction of approximately half of the successive intervals would be in the direction opposite to that found in the original melody. As a result, three sources of relational information would be distorted: successive intervals in terms of tone height, abstract intervals in terms of tone chroma, and the melodic contour as a pattern of pitch changes across a melody. Consider, for example, the interval $\mathrm{C}_{4}$ to $\mathrm{D}_{4}$, occurring in the original melody. Transformation of this interval into $C_{4}-D_{5}$ would distort the magnitude of the interval in terms of tone height, but would preserve the abstract interval in terms of tone chroma. The 2-semitone increase from $C_{4}$ to $D_{4}$ would be transformed into a 14-semitone increase from $C_{4}$ to $D_{5}$, both of these intervals being represented as an ascending second in the abstract octave. In contrast, transformation of $C_{4}-D_{4}$ into $C_{4}-D_{3}$ would violate not only the absolute interval, but also the abstract interval. The 2-semitone increase from $C_{4}$ to $D_{4}$ would be transformed into the 10 -semitone decrease from $C_{4}$ to $D_{3}$, which would be represented as a descending seventh in the abstract octave. At the same time, reversing the directions of half of the intervals in the melody would distort the contour of that melody.

In order to provide a fair test of a bidimensional model of pitch in melodic perception, it is necessary to displace the tones of a melody by octave intervals while preserving the melody's contour. Both tone height and absolute interval magnitude would then be violated, while tone chroma and melodic contour would be preserved, maintaining the abstract intervals. However, recognition of a melody could be 
facilitated by preservation of contour independent of any contribution of tone chroma, as contour alone may provide critical information for perception. This possibility is entirely reasonable from a musical standpoint, as forms such as the fugue often involve transformations which preserve melodic contour but distort interval relations (David \& Mendel, 1966). Moreover, empirical support for the role of contour has been obtained. Dowling and Fujitani (1971) found that subjects are quite accurate in identifying distorted melodies which preserve melodic contour, though their accuracy increases when successive intervals are preserved as well. Furthermore, melodic contour alone sometimes provides sufficient information for accurate perception. Dowling (1972), for example, has found that when melodies are subjected to various musical transformations, subjects are no more accurate in recognizing transformations which preserve exact interval relations than transformations which preserve only melodic contour. Similarly, Idson and Massaro (1976) have found that degraded tone patterns which can be uniquely specified by their contours are more accurately identified than patterns for which interval magnitude must also be perceived. Consequently, the contribution of contour alone must also be assessed.

The present research attempted to explore the utility of a bidimensional model of pitch for melodic perception. A melody recognition task was employed, in which the melodies were subjected to a variety of structural transformations designed to preserve or violate selective sources of information. The level of performance on these transformed melodies, with respect to the level of performance on the original untransformed melodies, provides an index of the importance of the manipulated information. To the extent that distortion of a source of information produces a performance decrement, that information can be thought to play a role in melodic perception. Similarly, if a given source of information can be eliminated without disrupting performance, then that information is not necessary for accurate melodic perception.

In the first experiment, five transformations were employed. Transformation O simply entailed playing the original melody, in order to provide a baseline for performance. Transformation OVC* replicated Deutsch's (1972b) manipulation. The component tones of the melody were displaced by octave intervals, violating the melodic contour by reversing the directions of approximately half of the successive intervals. In transformation OPC ${ }^{*}$, the component tones of the melodies were displaced by octave intervals, but now the melodic contour was preserved. Two additional transformations assessed the independent contribution of melodic contour by preserving the contour but violating all information as to absolute frequency and successive interval magnitude. These two transformations differed in terms of the precision with which the contour was maintained. In transformation PC, only the directions of the successive intervals were preserved. In transformation LT a linear transformation was taken of the contour, which halved the size of each of the component intervals. The resulting melodies would retain both the directions of the successive intervals and the relative magnitude of these changes. The unusually small intervals in these melodies should have no detrimental effects upon performance, as both Werner (cited by White, 1960) and White (1960) have found that once subjects obtain some practice with "microintervals" performance becomes quite accurate. Both of these contour-preserving transformations were included, since melody recognition studies (Dowling \& Fujitani, 1971; White, 1960) have shown that subjects are quite sensitive to preservation of relative degrees of contour.

A comparison of performance in identifying melodies under these five types of transformations should make it possible to evaluate the contributions of tone height, tone chroma, and melodic contour in melodic perception. Since transformation OVC* is identical to Deutsch's (1972b) manipulation, performance is expected to be quite poor. If tone chroma is ineffective in melodic perception, then performance on transformation OPC* should be equivalent to that on transformation OVC*. However, if octave convergence does occur under the octave displacement transformation, but perception is nevertheless disrupted due to violation of melodic contour, then performance on transformation OPC ${ }^{*}$ should be superior to transformation OVC*. Moreover, the contributions of octave convergence and melodic contour can be disambiguated by comparing performance on transformation OPC ${ }^{*}$ to that on transformations LT and PC. If any performance advantage seen for transformation OPC* over OVC* is due solely to preservation of melodic contour, then performance should be equally good on transformations OPC $^{*}$ and PC/LT. If further information is being provided by octave convergence, however, transformation OPC* should yield better performance than transformations PC/LT. A comparison of performance on transformations $\mathrm{O}$ and $\mathrm{OPC}^{*}$ allows an evaluation of the abstract perceptual octave proposed above. If this concept is valid, then performance on transformation OPC* shculd be comparable to that on transformation $O$, since the octave displacement manipulation preserving melodic contour maintains all of the intervals, in an abstract sense, which are found in the original melodies. To the extent that performance on transformation $\mathrm{O}$ is superior to that on transformation OPC*, absolute interval magnitude, rather than abstract interval identity, is play- 
ing an important role. Thus, the current study allows a direct evaluation of a bidimensional model of pitch in melodic recognition.

\section{EXPERIMENT 1}

\section{Method}

Subjects. The subjects were 25 University of Wisconsin students who received additional credit in an introductory psychology course for their participation. At the start of the experiment, the subjects were asked whether they knew all five melodies. Any subject who reported that he or she did not know one or more of the melodies was not continued in the study.

Apparatus. Four subjects could be tested simultaneously in separate sound-insulated rooms. All experimental events were controlled by a PDP-8L computer. The tonal stimuli were generated by a digitally controlled oscillator (Wavetek Model 155). The output of the oscillator was gated by two computer controlled audio switches (Iconic Model 0137) to separate amplifiers (McIntosh Model MC-50) for each ear. The tones were then presented over matched headphones (Grason-Stadler Model TDH-49). The visual display was presented over a digitally controlled array of light-emitting diodes (Monsanto Model MDA-III).

Design. The experimental design was a 2 by 5 by 5 factorial. The three factors were: the length of the melody, the identity of the melody, and the type of transformation performed upon the melody. The two lengths were either the first 8 or the first 12 notes of the melody. The five melodies were well known songs: Happy Birthday, London Bridge, Pop Goes the Weasel, On Top of Old Smokey, and God Rest Ye Merry Gentlemen. The five transformations $\left(\mathrm{O}, \mathrm{OPC}^{*}, \mathrm{OVC}^{*}, \mathrm{LT}\right.$, and $\left.\mathrm{PC}\right)$ involved changes in the frequencies of the component tones of the melodies, as described below.

Stimulus structure. The component tones of the melodies were 50 -msec sine waves, having 10 -msec rise/fall times. The intertone interval between successive tones was a constant 150 -msec interval. No rhythm information was present, and a sustained note was mimed by repeating the tone (see Dowling, 1973). The intensity of each tone was adjusted individually, in accord with the Robinson and Dadson (1956) equal-loudness contours. All of the melodies began on $\mathrm{A}$. For transformations $\mathrm{OPC}^{*}$ and $\mathrm{OVC}^{*}$, either $\mathrm{A}_{4}$ $(435 \mathrm{~Hz})$ or $A_{3}(870 \mathrm{~Hz})$ was used for the starting note. For transformations $O, P C$, and LT, the melody could be played in any one of the seven octaves $\left(A_{1}=54 \mathrm{~Hz}\right.$ to $\left.\mathrm{G}_{7}=6,570 \mathrm{~Hz}\right)$ spanned by the melodies in the cross-octave transformations. This insured that any differences which might be found between the within-octave $(\mathrm{O}, \mathrm{PC}, \mathrm{LT})$ transformations and the cross-octave (OPC*, OVC*) transformations wuuld not reflect simple differences in discriminability for tones in different octaves. This seems particularly important, as the difficulty of judging the direction of frequency shifts has been found to increase markedly for tones above about $4,000-5,000 \mathrm{~Hz}$ (Ward, 1970).

One instance of each of the five melodies was constructed for the four distorting transformations (OPC*, PC, LT, OVC*). These 20 melodies and 5 original melodies were used throughout the study, either the first 8 or the first 12 notes being played. The following heuristics were used to construct the melodies. In transformation $\mathrm{O}$, the original melodies were played. In transformations OPC* and $\mathrm{OVC}^{*}$, each tone was replaced by an exact octave multiple, with the replacement tone being drawn from the octave adjacent to that containing the immediately preceding tone. In transformation OPC* the contour of the original melody was preserved, so that the direction of each interval in the transformed melody was identical to the direction of the corresponding interval in the original melody. Both tones of a unison interval were always played in the same octave. In transformation OVC*, the contour of the melody was violated, by reversing the direction of approximately every other interval. Tones of a unison interval were played in different octaves, with half of the second tones shifted up an octave and half shifted down an octave. In addition, an attempt was made to insure that for each of the individual melodies, transformations OPC* and $\mathrm{OVC}^{*}$ spanned the same octave range and had approximately an equal distribution of notes in each octave.

In transformations PC and LT, the melodies were played entirely within a single octave, preserving the melodic contour while violating the absolute frequencies and successive interval magnitudes. In transformation PC, the component tones were chosen randomly, subject to the constraint that tones and intervals appearing in the original melody did not appear in the same position in the transformation. In transformation $L T$, the relative magnitudes of the intervals were preserved, by halving each interval. When the original interval involved an even number of semitones, halving that interval produced a new interval in which the second tone was not a note of the musical scale. The linear transformation insured that tones and intervals in the original melody would not appear in the same position in the transformed melody.

Procedure. The experiment was conducted on 2 consecutive days. Day 1 consisted of three sessions of 110 trials each, separated by 5 -min rest breaks. Day 2 consisted of four sessions of 110 trials each. The first 10 trials of each session were treated as unscored practice trials, though the subjects were not aware of this. On each trial, the subjects heard one of the five transformations of the five basic melodies. The subjects were to identify which of the five basic melodies was presented by pressing one of five buttons labeled with the names of the five melodies. The subjects were given a maximum response interval of $8 \mathrm{sec}$ from the onset of the melody. However, if all four subjects responded in less than this 8-sec interval, the experiment continued immediately upon the last response. Following the response interval, a .25 -sec visual presentation of a row of three asterisks $(* * *)$ was given over the lightemitting diodes to indicate the end of the response interval. A 1-sec intertrial interval was employed. All 50 ( 2 lengths $\times 5$ melodies $\times 5$ transformations) experimental conditions were completely random and occurred equally often within a session.

The same procedure was employed for all four experiments.

\section{Results}

The dependent measure is the percentage of correct responses. The percentage correct was calculated for each subject, in each session, at each length by melody by transformation condition. These percentages were then submitted to an analysis of variance on subjects, sessions, lengths, melodies, and transformations. The main effect of sessions was nonsignificant in the analysis $(F<1)$, as were all interactions involving sessions as a term. These results indicate that performance was not improving with increasing practice. Consequently, to increase the reliability of the individual scores, these data were pooled over sessions and reanalyzed. The same data analysis procedures were employed in all four experiments.

The longer melodies were identified approximately $6 \%$ more accurately than the shorter melodies $[F(1,24)=53.52, p<.001]$. However, all interactions involving melody length as a factor were nonsignificant in the analysis. This indicates that the length of the melody was affecting only the overall level of performance and was not differentially 
affecting either the individual melodies or the individual transformations. Consequently, the data will be discussed in terms of the average results for the two melody lengths.

Figure 2 presents the percentage of correct identifications of the melody as a function of the type of transformation performed on the melody. It is apparent from the figure that the type of transformation performed upon the melody had a large effect upon performance $[F(4,96)=85.79, p<.001]$. Displacing the component tones of the melodies by octave intervals in accord with the melodic contour (OPC*) yielded performance at essentially the same level as that observed on the original melodies $(\mathrm{O})$. A difference of only $3 \%$ was found between these transformations, and this was shown to be nonsignificant by a specific comparison. When the component tones of the melodies were displaced by octave intervals violating melodic contour, performance was substantially poorer than that observed for the original melodies. A performance decrement of $40 \%$ was found for transformation $\mathrm{OVC}^{*}$ relative to transformation $O[F(1,96)=420.57, p<.001]$.

The importance of melodic contour becomes even more apparent when the results on transformations LT and PC are considered. When only the pattern of pitch direction changes was preserved (PC), a $12 \%$ advantage was seen over transformation OVC* $[F(1,96)=34.16, p<.001]$. Performance on transformation $\mathrm{PC}$ was $27 \%$ poorer than on transformations OPC* and $\mathrm{O}[\mathrm{F}(1,96)=189.30, \mathrm{p}<.001]$. When a linear transformation (LT) of the contour was taken, a 7\% performance advantage was observed over a simple preservation of the contour (PC) $[F(1,96)=10.67, p<.005]$. This result supports the dual claim that it is meaningful to talk about preserving relative degrees of contour and that the processing system is able to effectively use any additional specificity of the contour. However, this additional information is still insufficient for melodic perception. A $20 \%$ performance advantage was found for the original melodies $(\mathrm{O})$ over those in transformation $\mathrm{LT}[\mathrm{F}(1,96)=99.73, \mathrm{p}<.001]$. This result argues that the equivalent performance observed on transformations $O$ and $O \mathrm{PC}^{*}$ is due to tone chroma, rather than an exact version of melodic contour.

Table 1 presents the percentage of correct identifications of the individual melodies, as a function of the type of transformation performed upon them. As can be seen from the table, the discriminability of the melodies differed $[\mathrm{F}(4,96)=2.94, \mathrm{p}<.005]$. The best performance was seen on God Rest Ye Merry Gentlemen (70\%), with a slightly lower level observed on Happy Birthday $(64 \%)$, London Bridge $(66 \%)$, and a still lower level on Pop Goes the Weasel

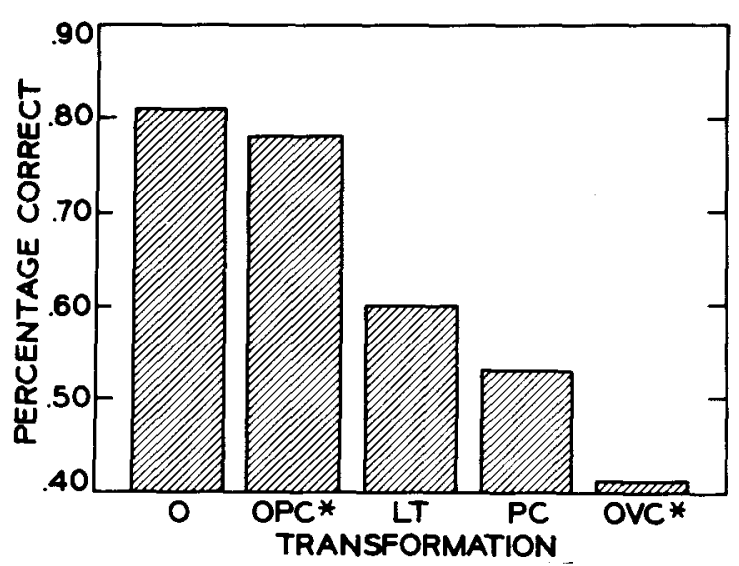

Figure 2. Percentage of correct identifications of the melody, as a function of the type of transformation performed upon the melodies (Experiment 1).

(59\%), and On Top of Old Smokey (55\%). A significant Melody by Transformation interaction was obtained $[\mathrm{F}(16,384)=13.06, \mathrm{p}<.001]$, though there was no consistent pattern to these differences.

The levels of performance in the group data were sufficiently high to suggest the possibility that ceiling effects might be obscuring possible differences between transformations $O$ and OPC*. Consequently, the subjects were divided into three groups on the basis of their level of performance on the original melodies. Figure 3 presents the percentage of correct identifications of the melody, as a function of the type of transformation, for each of these three groups of subjects. The top panel presents the average results for the four poorest subjects, whose identifications of the original melodies were at a level of between $41 \%$ and $50 \%$ correct. The middle panel shows the results for the 10 subjects whose performance was between $70 \%$ and $85 \%$ correct, while the bottom panel gives the data for the 11 subjects between $86 \%$ and $100 \%$ correct. It can be seen that subjects performing at quite different levels on the original melodies yielded essentially equivalent patterns of results, comparable to the pattern observed in the group data. Note, in particular, that even for the lowest performance group, essentially no difference was observed between transformations $O$ and OPC*, suggesting that ceiling effects are not responsible for the group results. The only exception to the replication of the group results for the individual subject groups was the finding, for the poorest group of subjects only, that performance was slightly better $(\mathbf{1 \% )}$ on transformation OVC* than on transformation PC. However, this most probably reflects a floor effect for these subjects. Chance, in this task, is $20 \%$ and performance on transformations PC and OVC* was at only $25 \%$ and $26 \%$, respectively. 
Table 1

Percentage of Correct Identifications of Each of the Individual Melodies as a Function of the Type of Transformation in Each of the Four Experiments

\begin{tabular}{|c|c|c|c|c|c|c|c|c|c|c|}
\hline \multirow[b]{2}{*}{ Melody } & \multicolumn{10}{|c|}{ Transformation } \\
\hline & $\mathrm{O}$ & $\mathrm{OPC}^{*}$ & LT & $\mathbf{L T}^{*}$ & PC & $\mathrm{PC}^{*}$ & $\mathrm{EC}^{*}$ & $\mathrm{CC}^{*}$ & ovc* & PI \\
\hline & \multicolumn{10}{|c|}{ Experiment 1} \\
\hline $\begin{array}{l}\text { Happy Birthday } \\
\text { London Bridge } \\
\text { Merry Gentlemen } \\
\text { Pop Goes the Weasel } \\
\text { Old Smokey }\end{array}$ & $\begin{array}{l}77 \\
89 \\
89 \\
85 \\
66\end{array}$ & $\begin{array}{l}70 \\
80 \\
86 \\
84 \\
72\end{array}$ & $\begin{array}{l}69 \\
69 \\
65 \\
53 \\
45\end{array}$ & & $\begin{array}{l}69 \\
52 \\
73 \\
44 \\
32\end{array}$ & & & & $\begin{array}{l}43 \\
39 \\
36 \\
31 \\
58\end{array}$ & \\
\hline & \multicolumn{10}{|c|}{ Experiment 2} \\
\hline $\begin{array}{l}\text { Happy Birthday } \\
\text { London Bridge } \\
\text { Merry Gentlemen } \\
\text { Pop Goes the Weasel } \\
\text { Old Smokey }\end{array}$ & $\begin{array}{l}97 \\
96 \\
76 \\
92 \\
80\end{array}$ & $\begin{array}{l}90 \\
93 \\
72 \\
94 \\
78\end{array}$ & $\begin{array}{l}87 \\
85 \\
69 \\
67 \\
55\end{array}$ & $\begin{array}{l}78 \\
83 \\
64 \\
60 \\
51\end{array}$ & $\begin{array}{l}67 \\
65 \\
49 \\
74 \\
69\end{array}$ & $\begin{array}{l}68 \\
61 \\
46 \\
62 \\
61\end{array}$ & & & $\begin{array}{l}42 \\
49 \\
46 \\
34 \\
62\end{array}$ & \\
\hline & \multicolumn{10}{|c|}{ Experiment 3} \\
\hline $\begin{array}{l}\text { Happy Birthday } \\
\text { London Bridge } \\
\text { Merry Gentlemen } \\
\text { Pop Goes the Weasel } \\
\text { Old Smokey }\end{array}$ & $\begin{array}{l}87 \\
96 \\
90 \\
88 \\
82\end{array}$ & $\begin{array}{l}79 \\
87 \\
79 \\
85 \\
86\end{array}$ & $\begin{array}{l}78 \\
88 \\
72 \\
54 \\
51\end{array}$ & & $\begin{array}{l}61 \\
71 \\
81 \\
61 \\
54\end{array}$ & & $\begin{array}{l}66 \\
73 \\
56 \\
67 \\
72\end{array}$ & $\begin{array}{l}54 \\
61 \\
67 \\
74 \\
74\end{array}$ & $\begin{array}{l}51 \\
45 \\
33 \\
32 \\
77\end{array}$ & \\
\hline & \multicolumn{10}{|c|}{ Experiment 4} \\
\hline $\begin{array}{l}\text { Happy Birthday } \\
\text { London Bridge } \\
\text { Merry Gentlemen } \\
\text { Pop Goes the Weasel } \\
\text { Old Smokey }\end{array}$ & $\begin{array}{l}78 \\
93 \\
81 \\
91 \\
89\end{array}$ & $\begin{array}{l}74 \\
88 \\
79 \\
89 \\
76\end{array}$ & $\begin{array}{l}71 \\
74 \\
64 \\
75 \\
65\end{array}$ & & $\begin{array}{l}66 \\
62 \\
65 \\
76 \\
57\end{array}$ & & & & $\begin{array}{l}42 \\
52 \\
47 \\
47 \\
36\end{array}$ & $\begin{array}{l}26 \\
30 \\
46 \\
05 \\
29\end{array}$ \\
\hline
\end{tabular}

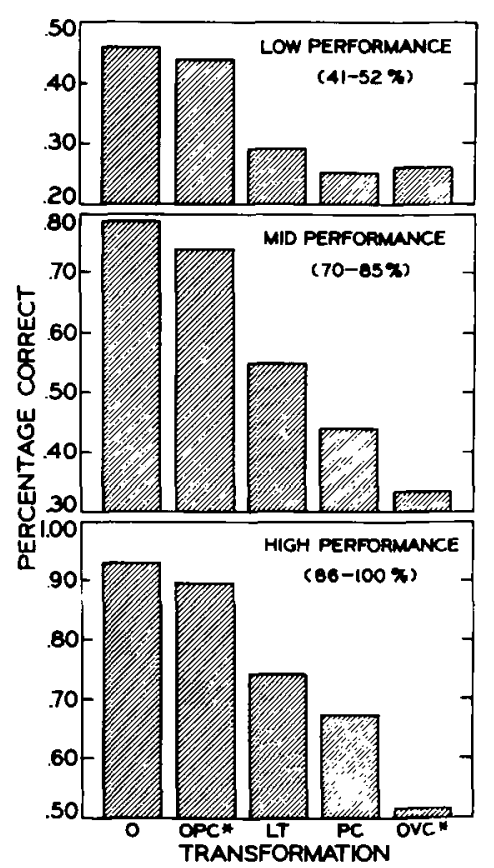

Figure 3. Percentage of correct identifications of the melody, as a function of the type of transformation, for three sets of subjects grouped on the basis of their overall levels of performance on the original melodies (Experiment 1).

\section{Discussion}

The results of the current study argue for the applicability of a bidimensional model of pitch to the perception of melodies by supporting the utility of the dimension of tone chroma. If chroma is functional in melodic perception, then tones which stand in an octave relationship should show unique generalization effects, such that the component tones of a melody can be displaced by octave multiples without disrupting perception. Direct confirmation of this prediction can be found in a comparison of performance under transformations $\mathrm{O}$ and $\mathrm{OPC}^{*}$ of the current study. In transformation OPC*, the component tones of the melodies were displaced by octave intervals preserving the melodic contour. Subjects identified these transformed melodies as accurately as they identified the original melodies (transformation $\mathrm{O})$. Yet, for the chroma dimension to be useful, contour must also be preserved. Though the only difference between transformations $O \mathrm{OP}^{*}$ and $O \mathrm{OVC}^{*}$ was the preservation of contour in OPC ${ }^{*}$, performance was substantially poorer on $\mathrm{OVC}^{*}$ than on OPC*. The better-than-chance performance on OVC* most likely resulted from preservation of half of the successive intervals of the original melody, providing partial information as to melody identity. However, the high level of performance seen on 
transformation OPC* cannot be due solely to preservation of melodic contour, as a large performance decrement was observed when only contour was available in both transformation PC and transformation LT.

The current results contradict Deutsch's (1969) model by demonstrating that the chroma dimension is functional in melodic perception. One possibility does exist for reconciling the results of transformation OPC ${ }^{*}$ with her model. The argument has been made (Deutsch, 1972b, Note 1) that while tone chroma cannot be used directly in recognizing a melody, it can be used indirectly to confirm a specific hypothesis about the identity of a melody. Subjects in Deutsch's (1972b) study were unable to generate hypotheses, since they heard the melody only once and had to identify it from the set of all possible melodies. In contrast, our subjects, who worked with a small set of melodies for hundreds of trials, could presumably have generated and tested hypotheses, accounting for the excellent performance on transformation OPC*. However, Deutsch's potential explanation is inadequate, since the hypothesis-testing strategy could equally have been employed in transformation OVC*, where performance was quite poor. The more probable explanation is that by disrupting contour, Deutsch (1972b) eliminated the utility of tone chroma. The present results demonstrate that, when contour is preserved, identification of a melody on the basis of tone chroma is possible, but with contour violated, it is not. This conclusion is strengthened by the internal replication of Deutsch's results in transformation OVC*, which suggests that the accurate performance on transformation OPC* cannot be due to procedural differences between the two studies.

After the present studies were completed, Dowling and Hollombe (1977, Experiment 2) reported evidence relevant to the possible occurrence of octave convergence when the contour of a melody is preserved. A melody recognition task was employed in which the subjects were to identify which of 10 familiar melodies was presented on each trial. The first 32 quarter-notes of the melody were presented at a rate of 1.33 notes/sec. Three experimental conditions were employed. The melodies were either played entirely within a single octave, with the component displaced randomly across octaves, or with the tones displaced across octaves but with contour preserved. Although large differences were found between the 10 melodies, more accurate identifications were obtained when contour was preserved than when contour was violated. However, even with contour preserved, performance was far less accurate when the tones of the melodies were displaced by octave intervals than when the tones were played entirely within a single octave. The Dowling and Hollombe results appear to contradict the present findings.
However, the preservation of contour in their study actually preserved only segments of the contour, since the tones of a unison interval were played in different octaves. For example, the interval $C_{4}-C_{4}$ could become $\mathrm{C}_{5}-\mathrm{C}_{4}$ or $\mathrm{C}_{3}-\mathrm{C}_{4}$. Thus, whenever the contour of a melody was flat (e.g., $C_{4}-C_{4}$ ), their manipulation introduced either a descending $\left(C_{5}-C_{4}\right)$ or an ascending $\left(\mathrm{C}_{3}-\mathrm{C}_{4}\right)$ interval. Dependent upon the number of unisons in a melody, this manipulation could radically disrupt the contour, greatly decreasing its utility.

The results of the first experiment argue that tone chroma is functional in melodic perception. Yet, the strength of this conclusion is somewhat minimized by the possibility that the excellent performance observed on transformation OPC* was due solely to the preservation of contour, independent of any contribution from tone chroma. The performance advantage on transformation OPC ${ }^{*}$, with respect to transformations LT and PC, rules out any simple explanation in terms of contour preservation. However, a somewhat more complex explanation still exists. The argument has been made (Idson \& Massaro, 1976) that the discriminability of the contour of a melody will increase with the size of the changes in frequency. By displacing the tones of the melodies in transformation OPC* across octaves, the magnitude of most frequency changes was doubled, effectively stretching out the contour. It is possible that the superior performance observed on transformation OPC* over transformations LT and PC was due to the more discriminable contour. In this case, no conclusions concerning tone chroma would be justified.

Experiment 2 was designed to explore this "contourstretching" hypothesis, by utilizing a transformation in which the contour is as discriminable as that in transformation OPC* but chroma is not preserved. This was accomplished by taking the melodies in transformations $\mathrm{LT}$ and $\mathrm{PC}$ and displacing their component tones by octave intervals, preserving the melodic contour. If the contour-stretching hypothesis is correct, then performance on these cross-octave contour-preserving transformations should be at a level equivalent to that seen for transformation OPC*. If chroma is critical, performance should be at a lower level than that observed on transformation OPC*. The strongest prediction which can be made from a bidimensional model is that the within-octave contour-preserving transformations (PC and LT) should yield performance at the same level as their cross-octave counterparts ( $\mathrm{LT}^{*}$ and $\mathrm{PC}^{*}$ ).

\section{EXPERIMENT 2}

\author{
Method \\ Subjects. The subjects were 18 University of Wisconsin under- \\ graduates who received credit towards an introductory psychology
}


course for their participation. None of the subjects had participated in Experiment 1.

Design. The experimental design was a 2 by 5 by 7 factorial. The three factors were melody length, melody identity, and type of transformation. The same two melody lengths, 8 or 12 notes, used in Experiment 1 were employed. The same melodies used in Experiment 1 were also employed: Happy Birthday, London Bridge, God Rest Ye Merry Gentlemen, Pop Goes the Weasel, and On Top of Old Smokey. Seven transformations of the melodies were now employed. The five basic transformations were retained from Experiment $1\left(\mathrm{O}, \mathrm{OPC}^{*}, \mathrm{PC}, \mathrm{LT}, \mathrm{OVC}^{*}\right)$ and two new transformations added ( $\mathrm{LT}^{*}$ and $\left.\mathrm{PC}^{*}\right)$.

Stimulus structure. The five transformations retained from Experiment 1 were constructed in the same way as in the earlier study. Transformations $\mathrm{LT}^{*}$ and $\mathrm{PC}^{*}$ were constructed by using the following algorithm. The melodies of both within-octave transformations were translated into cross-octave transformations by displacing the component tones of these melodies by octave intervals in accord with the melodic contour, in the same way that the original melodies were transformed in OPC*. Transformation $\mathrm{LT}^{*}$ represents the cross-octave version of $\mathrm{LT}$ and transformation $\mathrm{PC}^{*}$ represents the cross-octave version of $\mathrm{PC}$.

\section{Results}

The data analysis was identical to that of Experiment 1 . As the sessions factor again produced no significant effects, the data were pooled over sessions and reanalyzed. The main effect of melody length was nonsignificant in the analysis $(F<1)$, as were all effects involving melody length as a term. Consequently, the results given below are the average effects across the two melody lengths.

Figure 4 presents the percentage of correct identifications of the melody, as a function of the type of transformation. The type of transformation again had a large effect upon performance $[F(6,102)=$ $62.92, \mathrm{p}<.001]$. Replicating the earlier results, a difference of only $3 \%$ was found between transformations $\mathrm{O}$ and OPC*. A specific comparison between these conditions revealed this difference to be nonsignificant. Once again, a substantial decrement was found for transformation $\mathrm{OVC}^{*}$ relative to transformation $O[F(1,102)=255.33, p<.001]$. Performance was significantly poorer on transformation LT than on transformations $\mathrm{O}$ or OPC $[\mathbf{F}(1,102)=$ $40.76, \mathrm{p}<.001]$. Transformation LT again produced better performance than $\mathrm{PC}$, which was, in turn, substantially better than OVC*. Of primary interest, comparable levels of performance were found for transformations $\mathrm{LT}$ and $\mathrm{PC}$ and their respective cross-octave counterparts, transformations LT* and $\mathrm{PC}^{*}$. A $5 \%$ performance advantage was foửind for transformation $\mathrm{PC}$ over transformation. $\mathrm{PC}^{*}$ and a $6 \%$ advantage of transformation LT overtransformation $\mathrm{LT}^{*}$. Specific comparisons of transformation LT against transformation $\mathrm{LT}^{*}$ and trensformation PC against transformation PC* werevotis nonsignificant.

The percentage of correct identifications of each of the individual melodies, as a function the type of transformation, is shown in Intwit The

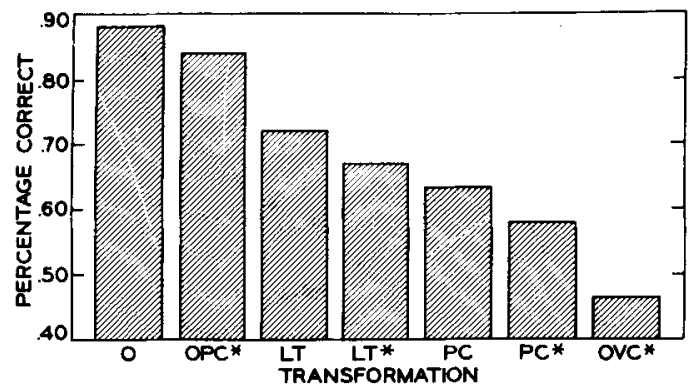

Figure 4. Percentage of correct identifications of the melody, as a function of the type of transformations performed upon the melodies (Experiment 2).

main effect of melody identity was significant in the analysis $[F(4,68)=4.76, p<.005]$. The overall levels of performance for the individual melodies did not reflect those found in Experiment 1. The highest levels of performance were seen on Happy Birthday (75\%) and London Bridge (76\%), with slightly lower levels of performance found for Pop Goes the Weasel $(70 \%)$ and On Top of Old Smokey (65\%) with the lowest performance seen on God Rest Ye Merry Gentlemen $(60 \%)$. The Melody by Transformation interaction was also significant $[F(24,408)=6.28, p<.001]$. However, the pattern of results did not reflect that observed in Experiment 1, suggesting that the interactions are not due to any consistent underlying effects.

\section{Discussion}

The results of Experiment 2 replicate exactly those of Experiment 1, with respect to the five overlapping transformations: O, OPC*, LT, PC, and OPC*. The results of fundamental importance concern performance on transformations $\mathrm{LT}^{*}$ and $\mathrm{PC}^{*}$, in which the melodies in transformations LT and PC were altered by dispersing the component tones across octaves in accord with the contour. This effectively stretched out the contours of the melodies in transformations $\mathbf{L T}^{*}$ and $\mathrm{PC}^{*}$ in a manner analogous to that for transformation OPC*. If chroma plays no role and the high level of performance seen on transformation OPC* is due to the increased discriminability of the contour, then performance on transformations LT $^{*}$ and PC* should have been at an equally high level. This result was not obtained; performance on transformations $\mathrm{LT}^{*}$ and $\mathrm{PC}^{*}$ was at a substantially lower level than performance on transformation OPC*. The levels of performance obtained on transformatons $\mathrm{LT}^{*}$ and $\mathrm{PC}^{*}$ did not differ significantly from the levels of performance obtained on their withinoctave counterparts, transformations LT and PC. This result suggests that the chroma dimension made the melodies in transformations LT* and PC* identifiably equivalent to those in transformations $\mathrm{LT}$ and 
PC. This is particularly interesting because halving the intervals in transformation LT resulted in tones in transformations LT and LT* which were not notes of the musical scale. The equivalent performance in these two transformations suggests that the similarity of tones in an octave relationship is not limited to notes of the musical scale.

Experiment 2 offers clear support for a bidimensional model of pitch, by eliminating an explanation for the results of Experiment 1 which was based upon the perception of contour. Unfortunately, as very little is known about the perception of contour, a large set of alternative explanations could be developed. Rather than evaluating each of these interpretations individually, a more parsimonious approach would be to test the entire class of contourbased explanations against the bidimensional model. Any contour-based explanation would argue that the superior performance on transformation OPC ${ }^{*}$ is due to preserving, in some sense, an exact version of the contour of the original melodies of transformation $O$. In contrast, a chroma explanation would submit that it is the octave relationship between the tones of the melodies in transformations $O$ and OPC* which is responsible. A clear test between these alternatives is to alter each note of the melodies in transformation OPC* by one or two semitones. This manipulation effectively eliminates the utility of the chroma dimension while preserving a highly accurate version of the contour. If some contour explanation is correct, then performance on such a transformation should be quite good. Alternatively, if a chroma explanation is valid, performance on such a transformation should be no better than performance on other contour-preserving transformations.

Experiment 3 was designed to carry out this test of the class of contour explanations, by employing two new transformations which altered the OPC* melodies by either one or two semitones. In transformation $\mathrm{EC}^{*}$, the absolute magnitude of each interval was increased by one or two semitones, effectively expanding the contour. In transformation $\mathrm{CC}^{*}$, each interval was decreased by a comparable amount, effectively contracting the contour.

\section{EXPERIMENT 3}

\section{Method}

Subjects. The subjects were 16 University of Wisconsin undergraduates who received credit in an introductory psychology course for their participation. None of the subjects had participated in either Experiment 1 or Experiment 2.

Design. The experimental design was a 2 by 5 by 7 factorial on melody length, melody identity, and type of transformations. The length of the melodies were either the first 8 or the first 12 notes of the melody. The same five melodies used in the earlier studies were employed: Happy Birthday, London Bridge, God Rest Ye Merry Gentlemen, Pop Goes the Weasel, and On Top of Old Smokey. The seven transformations included the five basic transformations from Experiment $1\left(\mathrm{O}, \mathrm{OPC}^{*}, \mathrm{OVC}^{*}, \mathrm{LT}\right.$, and $\mathrm{PC}$ ) plus the two new transformations $\left(\mathrm{CC}^{*}\right.$ and $\left.\mathrm{EC}^{*}\right)$.

Stimulus structure. The stimuli in transformations $\mathrm{O}, \mathrm{OPC} *$, OVC*, LT, and PC were identical to those in the previous studies. In transformations $\mathrm{EC}^{*}$ and $\mathrm{CC}^{*}$, cross-octave versions of the original melodies were constructed just as for transformation OPC*. However, in these transformations, the intervals were either larger (transformation $\mathrm{EC}^{*}$ ) or smaller (transformation $\mathrm{CC}^{*}$ ) than those of transformation OPC*. In transformation $\mathrm{CC}^{*}$ the absolute magnitude of each interval was decreased by either one or two semitones. These transformed melodies had intervals corresponding to the correct absolute interval +10 or 11 semitones, rather than an exact 12 -semitone octave. In transformation $\mathrm{EC}^{*}$, each interval was increased by one or two semitones so that the transformed melodies had intervals corresponding to the correct absolute interval +13 or 14 semitones rather than an exact octave.

\section{Results}

The data analysis was equivalent to that of Experiment 1 . The main effect of sessions was nonsignificant $(F<1)$, as were all interactions involving sessions as a term. Consequently, the data were pooled over sessions and reanalyzed. Since no overall difference was found between the two melody lengths, and since this factor did not interact significantly with any other factor, the data will be discussed in terms of the average results for the two melody lengths.

Figure 5 presents the percentage of correct transformations of the melody, as a function of the type of transformations performed upon the melodies. It is apparent from the figure that the type of transformation had a large effect upon performance $[\mathrm{F}(6,84)=49.94, \mathrm{p}<.001]$. The original melodies were identified $5 \%$ better than those of transformation OPC*; a difference which was marginally significant $[\mathrm{F}(1,84)=4.86, \mathrm{p}<.05]$. Replicating the earlier studies, transformation OVC* produced substantially poorer performance, yielding a performance

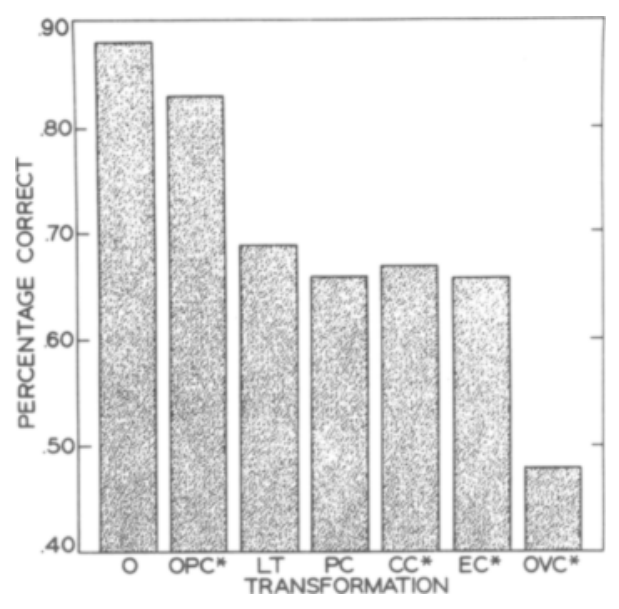

Figure 5. Percentage of correct identifications of the melody, as a function of the type of transformations performed upon the melodies (Experiment 3). 
decrement of $41 \%$ with respect to the original melodies $[\mathrm{F}(1,84)=287.61, \mathrm{p}<.001]$. As in the earlier studies, a lower level of performance was found on transformations $\mathrm{LT}$ and PC than that observed on either the original melodies or the melodies of transformation OPC*, while still being substantially better than performance on transformation OVC*.

The critical results concern performance on transformations $\mathrm{CC}^{*}$ and $\mathrm{EC}^{*}$. Both of these transformations yielded performance which was substantially poorer than that observed on the original melodies. Melodies in transformation EC* were identified $23 \%$ more poorly than the original melodies $[F(1,84)=$ $87.17, \mathrm{p}<.001]$, while a difference of $22 \%$ was found for the melodies of transformation EC* $[F(1,84)=78.89, p<.001]$. Performance on transformations $\mathrm{CC}^{*}$ and $\mathrm{EC}^{*}$ was at approximately the same level as that seen on the other contour-preserving transformations, LT and PC. The greatest difference in accuracy within this set of four transformations was between LT and PC, a 4\% difference. Performance on all four of the transformations which preserved only contour was substantially poorer than performance on the transformations which preserved both contour and chroma $\left(\mathrm{OPC}^{*}\right)[\mathrm{F}(1,84)=185.20$, $p<.001]$. At the same time, performance on the contour-preserving transformations was much better than that on the transformation preserving only chroma $\left(\mathrm{OVC}^{*}\right)[\mathrm{F}(1,84)=252.75, \mathrm{p}<.001]$. These results support the earlier conclusions that both chroma and contour are critical to melodic perception.

Unlike the earlier studies, no significant difference was found between the five individual melodies $(F<1)$, though a significant interaction was found between this factor and the type of transformation $[F(24,336)=9.98, p<.001]$. The three-way interaction of Melody by Transformation by Length was also significant $[F(24,336)=2.02, p<.005]$. There was no consistent pattern to these interactions, however. The results for the individual melodies as a function of the type of transformation are shown in Table 1.

\section{Discussion}

The results of Experiment 3 replicated those of the earlier studies for the five common transformations. The results of primary interest concern transformations $\mathrm{EC}^{*}$ and $\mathrm{CC}^{*}$. These results dramatically support the predictions of a bidimensional model, while disconfirming the entire class of contour-based explanations. Altering the melodies of transformation OPC* by only one or two semitones preserved a similar version of the contour, but eliminated the utility of the chroma dimension. Under these conditions, performance was at essentially the same level as that observed on transformations LT and PC.
These results provide exceedingly strong support for the interpretation that the high levels of performance seen on transformation OPC ${ }^{*}$ are due to the use of the chroma dimension.

The results of the first three studies indicate that a bidimensional model of pitch is applicable to the perception of melodies, provided that melodic contour is preserved. The role which has been attributed to contour is that of allowing the appropriate abstract intervals to be specified along the chroma dimension. This idea is supported by the finding that preservation of both chroma and contour yields superior performance to preservation of contour alone. The question must now be raised of whether contour plays any role beyond specifying abstract interval identity. It is possible that the performance advantage observed for transformation OPC* is due solely to the preservation of abstract intervals, independent of any unique contribution of melodic contour. To evaluate this possibility, a new transformation (PI) was employed in which the absolute magnitude of each interval was preserved, but its direction was reversed. This manipulation alters both frequency and contour, but maintains interval magnitude. For example, the interval $C_{4}-G_{4}$ would become $C_{4}-F_{3}$, substituting a descending fifth for an ascending fifth. If the advantage of transformation OPC* is due solely to the preservation of abstract interval magnitude, then performance on transformation PI should be as good as that on transformation OPC*. Alternatively, if contour, as a pattern of pitch changes, provides the advantage for transformation OPC*, then quite poor performance should be observed on transformation PI.

\section{EXPERIMENT 4}

\section{Method}

Subjects. The subjects were 22 University of Wisconsin undergraduates, who volunteered their participation in order to receive extra credit in an introductory psychology course. None of the subjects had participated in any of the preceding studies.

Design. The experimental design was a 2 by 5 by 6 factorial. The factors were again melody length, melody identity, and type of transformation. Melody lengths of 12 and 16 notes were used. The same five melodies employed in the previous experiments were again used: Happy Birthday, London Bridge, God Rest Ye Merry Gentlemen, Pop Goes the Weasel, and On Top of Old Smokey. The six transformations of the melodies included the five transformations employed previously (O, OPC*, OVC*, PC, and LT) and the new transformation (PI).

Stimulus structure. The stimuli in transformations $O, O P C^{*}$, $\mathrm{OVC}^{*}, \mathrm{PC}$, and LT were identical to those used in Experiments 1-3. The following heuristic was used to compute the melodies in transformation PI. The magnitude of each interval was calculated in terms of the number of semitones spanned by the interval. The melody was then restructured so that all of the interval magnitudes were preserved from the original melodies, but the direction of each interval was inverted. 


\section{Results}

The data analysis was identical to that of Experiment 1 . The main effect of sessions was nonsignificant in the analysis $(F<1)$, as were all interactions involving sessions as a term. Consequently, the data were pooled over sessions and reanalyzed.

The main effect of melody length was significant in the analysis $[F(1,21)=18.97, p<.001]$. The Length by Melody interaction was also marginally significant $[\mathrm{F}(4,84)=2.92, \mathrm{p}<.05]$. The shorter versions of On Top of Old Smokey were identified more accurately, while in all other cases more accurate recognition occurred for the longer melodies. However, both the Transformation by Length interaction and the Length by Transformation by Melody interaction $(F<1)$ were nonsignificant in the analysis. The data for the effect of the type of transformation will thus be presented averaged over the two lengths.

Figure 6 presents the percentage of correct responses, as a function of the type of transformation. The main effect of type of transformation was again highly significant $[F(5,105)=190.22$, $\mathrm{p}<.0011$. In the main, the results for the transformations carried over from the earlier studies were replicated. A difference of only $5 \%$ was obtained between transformations $O$ and $O \mathrm{OPC}^{*}$, which was shown to be nonsignificant by a specific comparison. As in the earlier studies, substantially poorer performance was found on transformation OVC* than on the original melodies $[\mathrm{F}(1,105)=143.13, \mathrm{p}<$ $.001]$. With respect to transformation OVC*, a $20 \%$ advantage was seen for transformation $\mathrm{PC}$ and a $25 \%$ advantage for transformation LT. However, neither of these contour-preserving transformations produced performance as accurate as that seen on transformation OPC $*$, a decrement of $11 \%$ occurring for transformation LT, and a decrement of $16 \%$ occurring for transformation PC. The difference between transformations LT and PC was not statistically significant. Of key importance, the poorest performance was found on transformation PI. A $60 \%$ performance decrement was observed for transformation PI relative to the original melodies, and a $55 \%$ decrement was observed relative to transformation OPC*.

Table 1 again presents the percentage of correct identifications of each of the individual melodies, as a function of the type of transformation. In contrast to the earlier studies, the individual melodies did not differ greatly in terms of their overall discriminability, and the main effect of melody identity was nonsignificant in the analysis: Happy Birthday (59\%), London Bridge (66\%), God Rest Ye Merry Gentlemen (63\%), Pop Goes the Weasel (63\%), and On Top of Old Smokey $(59 \%)$. However, the transformations did have differential effects upon the five melodies

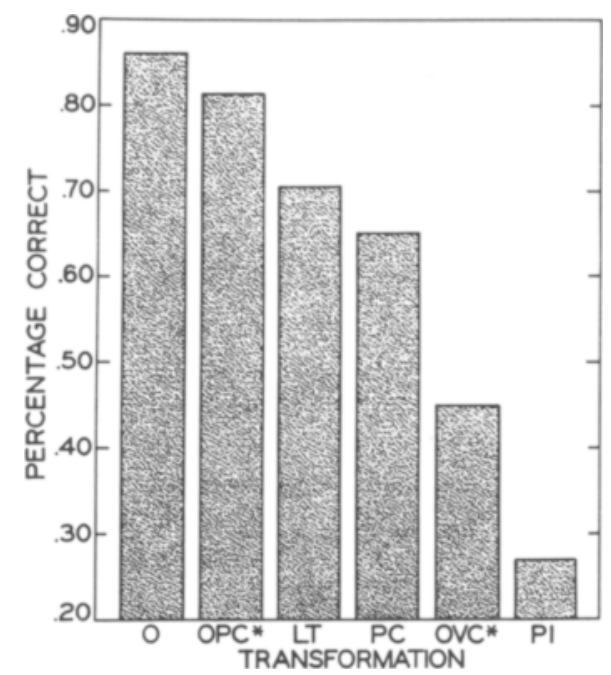

Figure 6. Percentage of correct identifications of the melody, as a function of the type of transformation (Experiment 4).

$[F(20,420)=10.87, p<.001]$, but no consistent pattern of results was present in the differences between individual melodies.

\section{Discussion}

The results for the five common transformations were essentially replicated in Experiment 4 . The critical findings concern transformation PI, in which interval magnitude was preserved and interval direction violated. Performance on transformation PI was far inferior to performance on any of the other transformations. This result clearly disconfirms the hypothesis that the performance advantage observed for transformation OPC ${ }^{*}$ was due to the preservation of abstract interval magnitude alone. Instead, performance on transformation OPC* appears to reflect the dual contribution of tone chroma and melodic contour. The exceedingly poor performance on transformation PI suggests that unless melodic contour is preserved, interval magnitude cannot be effectively utilized in melody perception.

\section{GENERAL DISCUSSION}

The current series of experiments provides strong evidence that a bidimensional model of pitch is applicable to melodic perception. When the structure of a melody is preserved, the pitch information given by tone chroma can be utilized for accurate perception. This finding raises the question of the nature of the information provided by tone chroma, and the manner in which this information interacts with melodic contour. While a variety of alternatives are possible, this question can be answered quite parsimoniously within the framework of perception within an abstract octave as discussed in the introduction. 
The dimension of tone chroma may serve to localize the pitch of a tone within an abstract octave, by tagging the tone for its appropriate pitch class. For heuristic purposes, processing along the chroma dimension can be envisioned as mapping each tone into an abstract representation, with the same representation serving all tones separated by an integer number of octaves. If, for the sake of parsimony, only the musical scale is again considered, the abstract octave would consist of 12 representative tones, each corresponding to one of the 12 notes of the traditional musical scale. From the perspective of tone chroma, the octave to which a particular tone belongs would be irrelevant. $A_{4}, A_{5}$, and $A_{6}$ would all be equivalent, in the sense that they are all tokens of the type A.

The implications of such a conceptualization for single tones are quite straightforward. It would simply insure that tones which are at octave intervals would be highly similar, as they would share a common abstract representation. This is essentially what has been proposed by all bidimensional models and would account for the similarity effects which have been seen for single tones.

The more interesting implications concern melodic perception. If the dimension of tone chroma is viable, then each tone of a melody would be mapped into its chroma representation. The potential then exists for perception to occur within an abstract octave space. In this case, the interval perceived between two successive notes would be the interval between their chroma representations. For example, the intervals $C_{4}-D_{4}, C_{4}-D_{5}$, and $C_{4}-D_{6}$ would all be mapped into the interval C-D. This is not to argue that such a mapping is automatic or inevitable. The tone height dimension could clearly be used to discriminate between the three intervals given above. However, if the use of tone height would not lead to accurate perception, as is the case for cross-octave intervals, then chroma representations could be employed. Yet logically, chroma alone cannot be sufficient for melodic perception. An abstract octave would, by definition, need to be circular (see, for example, Balzano, Note 2). Only in this way could a 12-note perceptual space capture the fact that $\mathrm{C}$ is sometimes higher and sometimes lower than D. As a result, intervals computed between chroma representations would be directionless. For example, the ascending second $C_{4}-D_{4}$ and the descending seventh $C_{4}-D_{3}$ would both be mapped into the directionless interval C-D. The fundamental importance of contour thus becomes quite reasonable: only if the contour of a melody is available will the intervals retain their correct directions and appropriate identities. As explicated in the discussions of the individual experiments, such a model can account exactly for all of the present results.
A somewhat different issue with which such a model must cope concerns musical techniques which depend upon the tones of a melody being perceived as falling into different frequency ranges. The principal example of such a technique is counterpoint, in which two or more melodies are combined in accordance with a set of definite rules (Piston, 1947) to form a harmonious texture. Composers such as Bach have written counterpoint for a single instrument, in which melodies in two different frequency ranges are interleaved, yielding the subjective impression of two discrete melodies (Ortmann, 1926). The validity of the counterpoint effect has been demonstrated empirically (Bregman \& Campbell, 1971; Dowling, 1973; Idson \& Massaro, 1976; Miller \& Heise, 1950; van Noorden, 1975), suggesting its perceptual reality. In fact, the failure to elicit effects of tone chroma for melodic perception in previous studies has often been attributed (Dowling \& Hollombe, 1977; House, 1977) to such effects.

At first blush, it would appear this phenomenon cannot be easily reconciled with the concept of tone chroma. Mapping the component tones into their abstract representations should eliminate the perceptual independence which is theoretically granted by a frequency separation. However, this line of reasoning depends upon the implicit assumption that the perceptual processes used in perceiving a stimulus will be the same regardless of the nature of that stimulus. This assumption is questionable. At least since the time of the Gestalt psychologists (see Koffka, 1935), the argument has been made that a stimulus has an internal structure which will determine the manner of its perception. From this perspective, it might be hypothesized that the internal structure of a melody has a perceptual integrity which functions to hold the melody together. If this were the case, it seems not implausible that whether octave convergence or octave independence occurs will depend largely upon which of these processes will facilitate unifying the melodic sequence. In the current study, where it was necessary to perceive pitch relationships between octaves, analysis of tone chroma would be emphasized, resulting in perception within the abstract octave. In counterpoint, in which a separation of two melodies on the basis of frequency is necessary, analysis of tone chroma would be minimized and analysis of tone height emphasized.

Though such an explanation is obviously ad hoc, there is a certain amount of evidence in favor of it. First, Idson and Massaro (1976) have lent support to the notion that different perceptual processes may be employed for different stimuli, by demonstrating that single tones and auditory patterns may be processed quite differently. Second, empirical studies of the counterpoint effect have demonstrated that smaller frequency separations between two patterns 
are needed to produce perceptual independence when the patterns are melodies (Dowling, 1972) rather than random tone sequences (Bregman \& Campbell, 1971), and therefore have an internal coherence. Similarly, Heise and Miller (1951) found that the frequency at which a tone appeared to become independent of a context pattern was, in part, a function of the nature of the pattern. Third, Dowling (1973) has found that when subjects are aware of the identity of the target melody in a recognition task, their ability to recognize the melody increases greatly, without a comparable rise in erroneous identifications of other melodies as the target. All of these results suggest that the extent to which large frequency separations will disrupt perception may be a function of the internal structure of the sequence. Direct evidence for this suggestion has been provided by the finding (Idson, 1977) that the degree to which an auditory pattern, whose tones alternate between octaves, will split into separate sequences is entirely a function of the structure of the pattern. Thus, there is no necessary discrepancy between counterpoint effects and the notion of perception within an abstract octave; the structure of a pattern will determine which of these phenomena will be elicited. Though these ideas are advanced only tentatively, and will require much additional study, they seem capable of providing a consistent account of the present data and a synthesis of previous work on bidimensional models of pitch and melodic perception.

\section{REFERENCE NOTES}

1. Deutsch, D. Octave equivalence and the processing of melodic sequences. Paper presented at the Meeting of the Acoustical Society of America, San Diego, 1976.

2. Balzano, G.J. On the bases of similarity of musical intervals: A chronometric analysis. Paper presented at the Meeting of the Acoustical Society of America, University Park, Pennsylvania, 1977.

\section{REFERENCES}

Allen, D. Octave discriminability of musical and non-musical subjects. Psychonomic Science, 1967, 7, 412-422.

Attneave, F., \& Olson, R. K. Pitch as a medium: A new approach to psychophysical scaling. American Journal of Psychology, 1971, 84, 147-166.

BACHEM, A. Note on Neu's review of the literature on absolute pitch. Psychological Review, 1948, 45, 161-162.

BACHEM, A. Time factors in relative and absolute pitch determination. Journal of the Acoustical Society of America, 1954, 26, 751-753.

BARD, J. W. Memory for absolute pitch: Studies in psychology, In Titchner commemorative volume. Worchester, Mass; L. N. Wilson, 1971.

Blackwell, H. R., \& Schlosberg, H. Octave generalization, pitch discrimination, and loudness thresholds in the white rat. Journal of Experimental Psychology, 1943, 33, 407-419.
Bregman, A. S., \& Campbell, J. Primary auditory stream segregation, perception of order in rapid sequences of tones. Journal of Experimental Psychology, 1971, 89, 244-249.

DaVID, H. T., \& MENdEL, D. A Bach reader. New York: Norton Books, 1966.

De utsch, D. Music recognition. Psychological Review, 1969, 76. 300-307.

Deutsch, D. Tones and numbers: Specificity of interference in short-term memory. Science, 1970, 168, 1604-1605.

DEUTSCH, D. Effect of repetition of standard and comparison tones in recognition memory for pitch. Journal of Experimental Psychology, 1972, 93, 156-162. (a)

DEUTSCH, D. Octave generalization and tune recognition. Perception \& Psychophysics, 1972, 11, 411-412. (b)

DeUTSCH, D. Octave generalization of specific interference effects in memory for tonal pitch. Perception \& Psychophysics, 1973, 13, 271-275. (a)

DEUTSCH, D. Interference in memory between tones adjacent in the musical scale. Journal of Experimental Psychology, 1973, 100, 228-231. (b)

DEUTSCH, D. Generality of interference by tonal stimuli in recognition memory for pitch. Quarterly Journal of Experimental Psychology, 1974, 26. 229-234.

Dowling, W. J. Recognition of melodic transformations: Inversion, retrograde, and retrograde inversion. Perception \& Psychophysics, 1972, 12, 417-421.

Dowling, W. J. The perception of interleaved melodies. Cognitive Psychology, 1973, 5, 322-337.

Dowling, W. J., \& Fujitani, D. A. Contour, interval, and pitch recognition in memory for melodies. Journal of the Acoustical Society of America, 1971, 49, 524-531.

Dowling, W. J., \& Hollombe, A. W. The perception of melodies distorted by splitting into several octaves: Effects of increasing proximity and melodic contour. Perception \& Psychophysics, 1977, 21, 60-64.

Heise, G. A., \& Miller, G. A. An experimental study of auditory patterns. American Journal of Psychology, 1951, 64, 68-77.

House, W. J. Octave generalization and the identification of distorted melodies. Perception \& Psychophysics, 1977, 21, 586-589.

Hubel, D. H., \& WIEsel, T. N. Receptive fields, binocular interaction, and functional architecture in the cat's visual cortex. Journal of Physiology, 1962, 160, 106-154.

HUMPhREYs, L. G. Generalization as a function of method of reinforcement. Joumal of Experimental Psychology, 1939, 25, 361-372.

IDson, W. L. The perception of structure in auditory patterns. Unpublished doctoral dissertation, University of Wisconsin, 1977.

IDson, W. L., \& Massaro, D. W. Cross-octave masking of single tones and musical sequences: The effects of structure on auditory recognition. Perception \& Psychophysics, 1976, 19, $155-175$.

Koffka, K. Principles of Gestalt psychology. New York: Harcourt Brace, 1935.

MEYer, M. On the attributes of the sensations. Psychological Review, 1904, 11, 83-103.

Miller, G. A., \& Heise, G. A. The trill threshold. Journal of the Acoustical Society of America, 1950, 64, 637-638.

NeTrle, B. Music in primitive culture. Cambridge: Harvard University Press, 1956.

Ortmann, D. On the melodic relativity of tones. Psychological Monographs, 1926, 35, (Whole Number 165).

Piston, W. Counterpoint. New York: Norton, 1947.

RÉvész, G. An introduction to the psychology of music. Norman: Oklahoma University Press, 1954.

Roninson, D. W., \& DADson, R. S. A re-determination of the 
equal loudness relations for pure tones. British Journal of Applied Physics, 1956, 7, 166-181.

Ruckmick, C. A. A new classification of tonal qualities. Psychological Review, 1929, 36, 172-180.

SHEPARD, R. N. Circularity of judgments of relative pitch. Journal of the Acoustical Society of America, 1964, 36, 2346-2353.

STEVENS, S. S., \& Volkmann, J. The relation of pitch to frequency. American Journal of Psychology, 1940, 53, 329-353.

Stevens, S. S., Volkmann, J., \& Newmann, E. G. A scale for the measurement of the psychological magnitude of pitch. Journal of the Acoustical Society of America, 1937, 8, 185-190.

ToveY, D. F. The forms of music. New York: World Publishing, 1956.
VAN Noorden, L. P. A. S. Temporal coherence in the perception of tone sequences. Unpublished doctoral dissertation, Institute for Perception Research, Eindhoven, The Netherlands, 1975.

WARD, W. D. Musical perception. In J. V. Tobias (Ed.), Foundations of modem auditory theory (Vol. 1). New York: Academic Press, 1970.

WhITE, B. Recognition of distorted melodies. American Journal of Psychology, 1960, 73, 100-107.

(Received for publication January 18, 1978; revision accepted August 17, 1978.) 